\title{
SCIENTIFIC CCD PROSPECTS FOR 1994 AND BEYOND
}

\author{
Paul R. Jorden and A. P. Oates \\ Royal Greenwich Observatory
}

\begin{abstract}
We review the present status of CCD use at major observatories, discuss the various developments that are leading to improved sensors. The choice of devices is quite extensive, but far from stable! A look at progress over the last few years helps to put present and future developments in perspective. Several areas of active CCD development are presented and discussed.
\end{abstract}

\section{INTRODUCTION AND CURRENT STATUS OF OPTICAL CCDS.}

There is no doubt that for the last decade CCDs have been the principal optical sensor at most observatories. The number of manufacturers, and of devices has increased over this period, as illustrated in Table 1. The table lists a snapshot of the main CCD suppliers and indicates the primary sensors that have been in widespread use at each time period. Prototype, or uncommon types are not shown here.

TABLE 1

Principal CCD manufacturers, and devices, 1980-1994

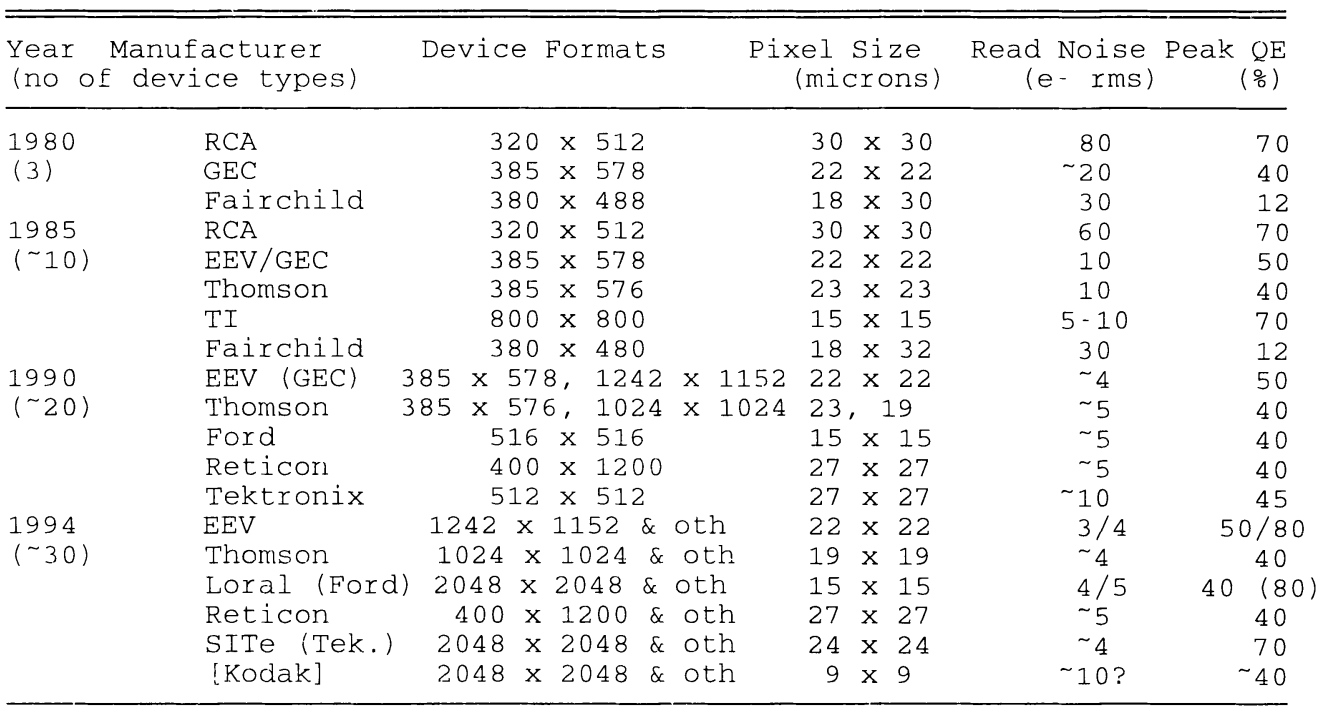


As a prelude to a discussion of future developments we shall review here the types of devices and performances that can be obtained today. The routine supply of good quality scientific CCDs is somewhat precarious, with a limited number of sources. Within the last few years the use of foundry manufacturers has developed - particularly with many devices having been made by Ford/Loral. Foundries offer the possibility of supplying a large quantity of devices at manageable costs; however, the yield and performance of such devices are not guaranteed.

The following tables present a broad picture of CCD usage by observatories around the world; quite a few different types of CCD are in use, obtained through several routes. Table 2 illustrates the wide range of devices known to be offered by major observatories. There may be a few omissions, but on the whole it should at least present a snapshot of current use. Most observatories are in the process of trying to upgrade to larger CCDs, so the picture could change quite a lot in the next year or so.

TABLE 2

Parameters of CCDs, used by major observatories (ESO, CTIO, KPNO, RGO, AAO).

\begin{tabular}{|c|c|c|c|c|c|c|}
\hline \multirow[t]{2}{*}{ CCD TYPE } & \multirow{2}{*}{$\begin{array}{l}\text { Pixel size } \\
\text { (Micron) }\end{array}$} & \multicolumn{2}{|c|}{ Format } & \multirow{2}{*}{\multicolumn{2}{|c|}{$\underset{X}{\text { Image }}$ size }} & \multirow[t]{2}{*}{ Status } \\
\hline & & $\mathrm{X}$ & Y & & & \\
\hline GEC P8603/ EEV-02-06 & 22.0 & 385 & 578 & 8.5 & 12.7 & Comm. \\
\hline RCA SID501 (\&640 x 1024) & 30.0 & 320 & 512 & 9.6 & 15.4 & Obs. \\
\hline $\mathrm{EEV} C \mathrm{CD}-05-30 \quad(\mathrm{P} 88300)$ & 22.5 & 1242 & 1152 & 27.9 & 25.9 & Comm. \\
\hline Tektronix TK512 (SITe) & 27.0 & 512 & 512 & 13.8 & 13.8 & Comm. \\
\hline Tektronix TK1024 (SITe) & 24.0 & 1024 & 1024 & 24.6 & $24 \cdot 6$ & Comm. \\
\hline Tektronix TK2048 (SITe) & 24.0 & 2048 & 2048 & 49.2 & 49.2 & Comm. \\
\hline Reticon RA1200 & 27.0 & 400 & 1200 & 10.8 & 32.4 & Comm. \\
\hline Thomson TH7896 (TH1024) & 19.0 & 1024 & 1024 & 19.5 & 19.5 & Comm . \\
\hline Loral (Ford) NOAO-3 k x $1 \mathrm{k}$ & 15.0 & 3072 & 1024 & 46.1 & 15.4 & Found \\
\hline Loral (Ford) SAO-Lick $2 \mathrm{k} \times 2 \mathrm{k}$ & 15.0 & 2048 & 2048 & 30.7 & 30.7 & Found \\
\hline $\mathrm{TI}-800$ & 15.0 & 800 & 800 & 12.0 & 12.0 & Obs. \\
\hline
\end{tabular}

As a further illustration of present-day status the Table 3 shows the principal parameters of one of the main camera types that we operate at the international Roque de los Muchachos Observatory on La Palma. This camera, utilizing the Tek1024 thinned CCD, exhibits performance that is typical of modern, high-grade sensors. We currently use CCDs for $94 \%$ of all observing on ING telescopes.

To conclude this section, Table 4 presents a list of most CCDs that are of interest for large-area (> $256 \times 256$ pixels) scientific imaging; the scene does change quite rapidly so it is unlikely to be an exhaustive list. The use of foundry sources for CCDs makes it particularly hard to be definitive about the exact number and type of devices that are around. Obsolete sensors (even if still in use) are not listed. Included in the table are many devices that are in prototype form, and not routinely available; some will become commercially available, some will not! Commercially available devices are shown with an *. 
TABLE 3

An example of CCD parameters - The ING TEK1 CCD.

\begin{tabular}{|c|c|}
\hline $\begin{array}{l}\text { Detector type } \\
\text { Format } \\
\text { Pixel size } \\
\text { Array size } \\
\text { Readout noise } \\
\text { Operating temperature } \\
\text { Dark current } \\
\text { Pixel sampling times } \\
\text { Quantum efficiency } \\
\text { Charge transfer efficiency } \\
\text { Cosmetics (grade-1 device) } \\
\text { Cosmic ray rate }\end{array}$ & 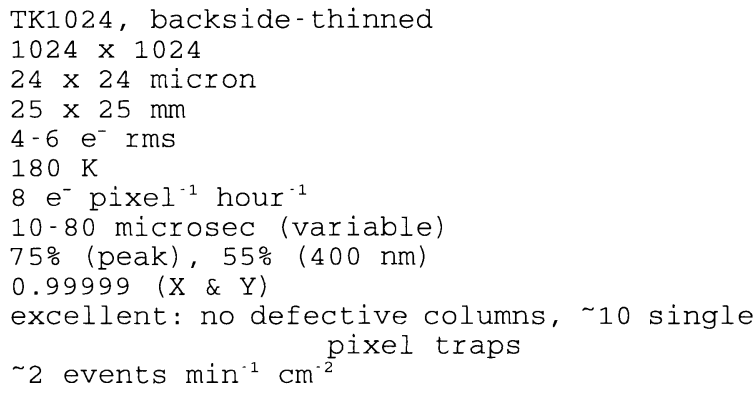 \\
\hline
\end{tabular}

2. AREAS OF ACTIVE DEVELOPMENT, AND PROJECTIONS INTO THE NEAR FUTURE.

In this section various areas of current activity and interest will be introduced. Since several topics are due to be presented in more detail elsewhere in this Symposium, only a limited discussion will be given here. A few interesting features, that some users may not be aware of, are presented and some specific new developments are highlighted.

\subsection{Mosaics - Multiple Chips, Multiple Outputs, Higher Speeds.}

The requirements of large focal plane areas are such no single CCD is big enough. The largest sensors presently available have a $2 \mathrm{k} \mathrm{x} 2 \mathrm{k}$ format; a $4 \mathrm{k}$ x $4 \mathrm{k}$ size appears unrealistic at the current state of technical development. A mosaic of modest sized CCDs is considered to be the realistic solution to this problem. There are several developments of $4 \mathrm{k} \times 2 \mathrm{k}$ format devices at present; most current designs envisage using $2 \mathrm{k} \mathrm{x} 4 \mathrm{k}$ three-edge buttable chips (a $V L T$ $C C D)$ to create large focal plane arrays.

This VLT CCD is currently planned for at least the following telescopes - Keck, Gemini, ESO-VLT, Subaru, as well as many smaller ones (RGO, AAO, Galileo etc.). Within the next year or so we should hope to see the successful introduction of at least some devices of this format, although we should not underestimate the difficulties of achieving high performance (eg Quantum Efficiency) from such large devices.

With the use of large-format devices it becomes more and more important to achieve higher speeds of readout, in order to minimise total frame readout times. All large-format devices have multiple outputs (two or four) to facilitate multiple channel readout.

With improved baseline readout noise performance, it is possible to increase the speeds of readout and still achieve low readout noise. Whereas $10 \mathrm{e}^{-}$was about the lowest possible readout noise a decade ago, it is now possible to achieve $\sim 2 \mathrm{e}^{-}$noise (at $\sim 50 \mathrm{kHz}$ pixel rates), and $<10 \mathrm{e}^{-}$is still achieved - but at much higher readout speeds than before. The paper by Luppino (1995, this Symposium) discusses such mosaics in more detail. 
TABLE 4

A selection of scientific CCDs (1994).

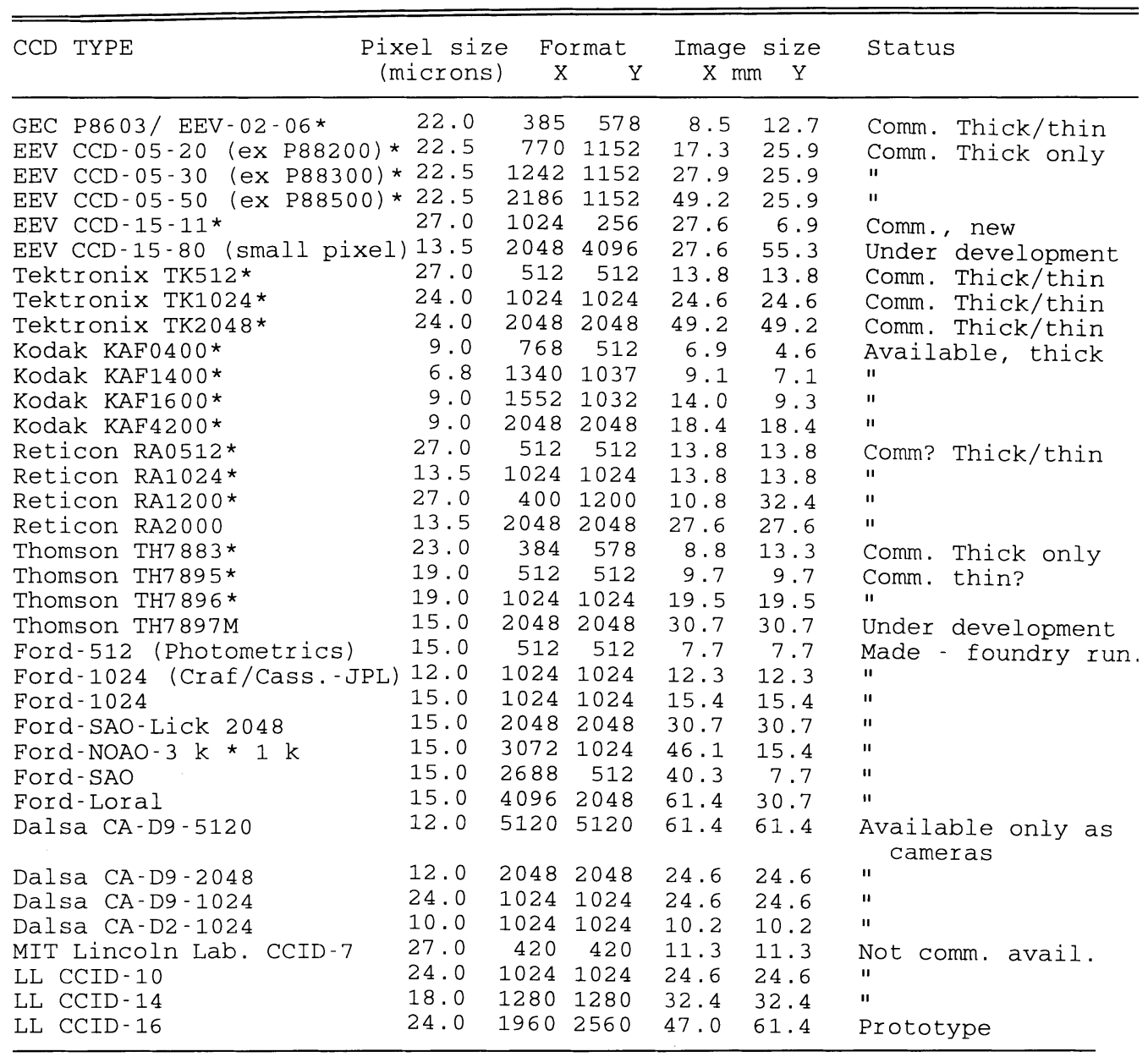

\subsection{Higher Speeds, Lower Noise and Adaptive Optics.}

A particular area of recent development has been that of low-noise at high speeds. Lincoln Labs have demonstrated that it is possible to make a CCD which yields a readout noise $\sim 6 / 7$ $\mathrm{e}^{-}$at one $\mathrm{MHz}$ pixel rates (Burke et al. 1991); this noise was previously only achieved at $50 \mathrm{kHz}$ pixel rates. By designing the output circuitry of the CCD to have a very high charge-to-voltage conversion factor it is possible to get a lower effective noise during readout - this is achieved at the expense of a more limited total charge-handling range. It is interesting to note that such a design can yield a one to two $\mathrm{e}^{-}$readout noise at normal slow-scan rates $(20-50 \mathrm{kHz})$. 
At present (for example the TK1024 device) a readout noise of four to five $\mathrm{e}^{-}$is routine, with somewhat lower figures being possible at very slow rates. It is now clear that noise figures of two to three $\mathrm{e}^{-}$ought to be common in the next few years, as the higher-gain amplifier designs are introduced. The high $\mathrm{QE}$ of CCDs, coupled with such low noise, means that traditional (intensified) photon-counting systems have little advantage for most common astronomical applications.

The main interest in higher speed CCDs has developed from the requirements of adaptive optics systems. Here, it is crucial to have a high sensitivity at high frame rates ( $\sim$ one $\mathrm{kHz})$. High sensitivity is achieved through the use of a backside-thinned device, together with low readout noise at this high speed. Several CCD suppliers are developing higher speed sensors these have a use in some industrial markets as well as for scientific imaging. For Adaptive Optics a thinned $64 \times 64$ format $\mathrm{CCD}$, with $\sim$ five $\mathrm{e}^{-}$noise at one $\mathrm{MHz}$ pixel rates is anticipated. The non-commercial Lincoln Laboratory has made such a device, and other manufacturers are expected (hoped?) to follow suit.

\subsection{Different Chip Designs - Larger Areas, Higher QE, Lower Noise, Smaller Pixels.}

Since the advent of foundry runs it has been possible for novel chips to be designed, built, and made available to the community (just). Examples of these include buttable-chips, multiple-output designs, and even the Geary polar-coordinate device (Geary and Luppino 1994). Ford-Aerospace (now Fairchild-Loral) made the first foundry CCDs for astronomy, and more recently the Orbit foundry has made similar devices (see Stover et al. 1995, this Symposium). This source of supply is now well established; however the disadvantages are that the yield is uncertain, and quality is not assured. Devices have to be thinned elsewhere in order to obtain a high quantum efficiency. With the uncertainty in supply of good large CCDs, several foundry sources are currently being investigated.

Higher quantum efficiency is always much in demand. The technique of manufacturing thinned, backside-illuminated devices is still proving difficult for all manufacturers. RCA supplied such devices in 1980, TI and Tektronix have supplied many such devices since then, and other manufacturers have developed thinning techniques. However, it is still proving difficult to make and supply large-area, high quality thinned devices. Mike Lesser has been particularly active in thinning chips from foundry sources. He has also demonstrated that very high QE (100\%) can be achieved - by selective AR-coating of the silicon at certain wavelengths (Lesser 1994). It is also feasible to optimize the coating for red or blue regions, depending on the instrumental application required. As an aside, an optimized blue coating means that the chip will have a finite red-wavelength reflection loss - which results in a significant degree of fringing at the longer wavelengths.

$\mathrm{CCD}$ designers and manufacturers have been able to reduce readout noise, mainly as a result of experience and empirical design. The output transistors on CCDs can now yield very low noise, such that four to five $\mathrm{e}^{-}$is often routinely attained from commercial chips. Noise figures of two to three $\mathrm{e}^{-}$have been seen in some commercial chips and noise figures in the region of one to two $\mathrm{e}^{-}$appear possible with certain limitations. A novel skipper amplifier has been produced which can potentially reduce noise by multiple sampling (Chandler et al. 1990), but it seems to have little benefit now that the basic CCD noise levels can be reduced so much. 


\subsection{Lower Dark Current-MPP Devices and Wobble-Clocks.}

Many CCDs are now available in MPP (multi-phase-pinned) form; this allows a reduction of dark current by about a factor of 100 . The devices require operation in a slightly different manner, and have a somewhat lower full-well capacity. The use of MPP devices allows the possibility of operating at higher temperatures than otherwise possible. In the case of cryogenic systems, a higher operating temperature can yield a better red-response. In other cases it can allow Peltier rather than cryogenic cooling, or even no cooling at all. It is also possible to minimise dark current by modulating CCD clocks during the exposure period (Thorne et al. 1990, Burke and Gajar 1991); this technique is particularly beneficial for non MPP devices

\subsection{Improved Red Response; Deep-Depletion Devices.}

Some devices have been made with a higher resistivity silicon (eg $1000 \mathrm{ohm}-\mathrm{cm}$, c.f. the normal $20-50 \mathrm{ohm}-\mathrm{cm}$ ). This gives a deeper depletion region within the device. If the device is manufactured with a thicker active region this allows it to have a greater absorption of near-infrared wavelengths. The quantum efficiency can be increased at wavelengths longer than about $700 \mathrm{~nm}$; it also has benefits for x-ray charge detection (for example see Holland et al. 1992).

\subsection{Antiblooming.}

CCD imagers traditionally suffer from blooming or image spread when over-exposed. TV-camera sensors are often built with internal antiblooming structures, but this is not appropriate for scientific sensors. However, by modulating CCD clocks during the exposure period, it is possible to attenuate strongly or even eliminate the undesirable effects of pixel over-exposure; (see Neely and Janesick, 1993). The technique may need some care to implement, but could be quite powerful for selected astronomical applications. Of course, over-exposure of part of the CCD array can still lead to internal reflections and the risk of scattered light.

\subsection{Improved Electronics - More Compact, Lower Power, More Dynamic Range.}

The improvements in CCD design need to be matched by improvements in electronics systems. There are three main areas requiring development:

a) Multi-channel systems required for faster parallel readouts.

b) Higher speed readout and data-transfer systems required - for cryogenic and adaptive-optics systems.

c) 18-bit A-D converters and electronic systems are needed to take advantage of the wide inherent dynamic range of CCDs. (An 18-bit (262144) range allows us to have $260 \mathrm{~K} \mathrm{e}^{-}$signal range, with a two $\mathrm{e}^{-}$readout noise, and one $\mathrm{e}^{-}$quantisation unit). Most systems (currently 16-bit), with a one $\mathrm{e}^{-}$digitization unit, only allow a full-range of $65536 \mathrm{e}^{-}$.

\section{RGO CCD ACTIVITIES.}

To conclude this review, we will take the opportunity to present a brief overview of current areas of RGO CCD development: 


\subsection{WYFFOS}

This is a multi-fiber spectrograph for the William Herschel Telescope (4.2-m) - it is due for completion at the end of 1994. It has a robotic fiber-positioner (AUTOFIB) at the prime focus; this feeds a custom-designed optimised spectrograph which resides at the Nasmyth (GHRIL) platform. The detector is a thinned Tek1024 CCD embedded in a Schmidt cryogenic camera. (See Bingham et al. 1994).

\subsection{INT PF Mosaic}

This project, in collaboration with US and Netherlands groups aims to provide a large-area CCD focal plane for the Isaac Newton Telescope (2.5-m). Four thinned Loral CCD chips will provide a $4 \mathrm{k} \mathrm{x} 4 \mathrm{k}$ mosaic format, covering a $25 \times 25$ arcmin FOV, with 0.37 arcsec pixels. A fifth co-planar CCD will be used to provide autoguiding for the telescope. An outline of the camera-head design is shown in Fig. 1; the whole instrument will be described fully in a future publication. The camera is to be used for surveys and supernovae searches, and is scheduled for completion at the end of 1995.

\subsection{Large-Format CCD Upgrades}

We are engaged in a program to enhance most of our CCD imaging facilities by providing larger, improved sensors. We have a batch of Loral $2 \mathrm{k} \mathrm{x} 2 \mathrm{k}$ devices that has been manufactured, and is currently undergoing thinning (by Mike Lesser). We have also just placed a contract (with EEV) for $4 \mathrm{k} \mathrm{x} 2 \mathrm{k}$ thinned CCDs. Our requirements are for larger areas, small pixels, lower noise, and good (thinned) quantum efficiency.

\subsection{Intra-Pixel Response Measurements}

We have recently analyzed the internal structure of CCDs in some detail (see Jorden et al. 1994). Several CCDs were tested in the laboratory to determine how response changes with intra-pixel sampling position. The internal architecture has an appreciable influence on the response of a CCD that have implications when the image in undersampled; this can be important in many areas of use - including photometry, spectral extraction, astrometry, centroiding (AO systems).

\section{5 $\mathrm{QE}$ as a function of temperature.}

We have analysed the effects of operating at different temperatures. Operating temperature is well known to influence the far-red response of a CCD, but we also found response changes at other wavelengths. This has been relevant recently in discussions about achieving good UV response - at ambient temperatures (where many manufacturers check the devices), and when cryogenically cooled (where we need to operate them). Fig. 2 shows spectral response curves (of ING telescope CCDs) that are typical of frontside and backside performance (at a cryogenic temperature). Fig. 3 shows measurements of A TK1024 thin CCD, and demonstrates the variation of $\mathrm{QE}$ with temperature from 173-293K. 


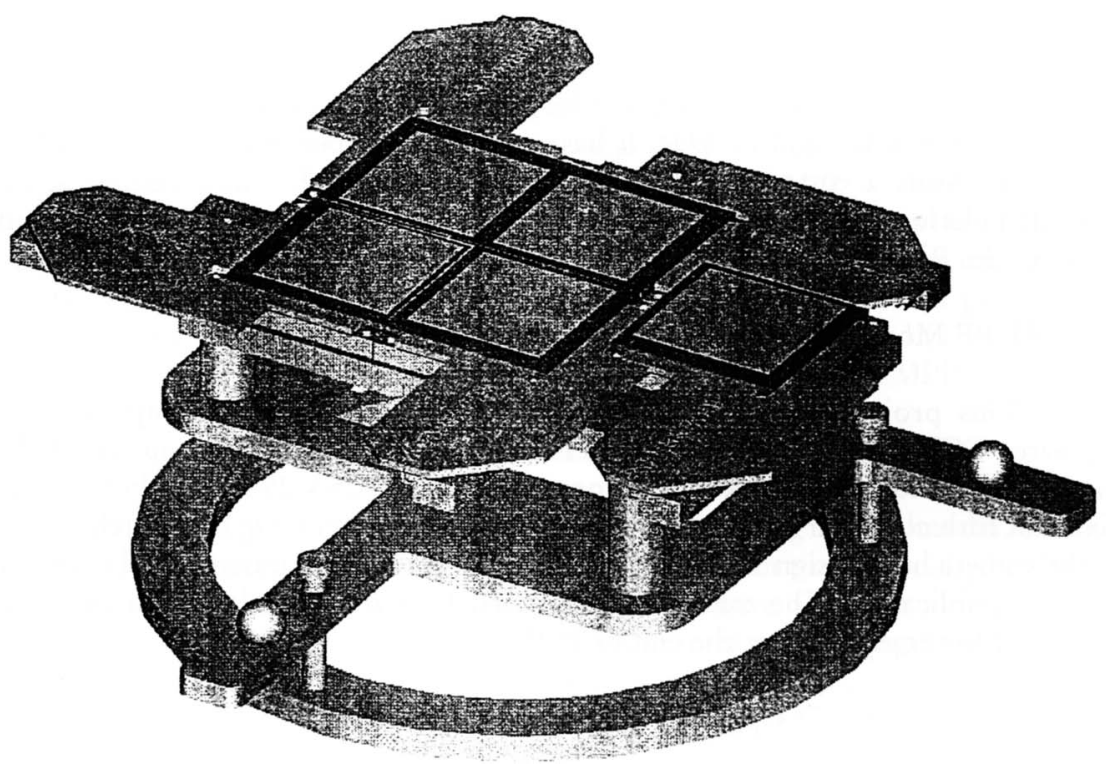

Fig. 1. The Isaac Newton Telescope Prime Focus Mosaic CCD camera.

3.6 Readout-Noise as a Function of Speed.

Prompted by an analysis of the requirements for a wavefront sensor, we have been operating CCDs at higher speeds than usual for cryogenic systems. Adaptive optics, and other wavefront sensors require much higher readout speeds than is normal in slow-scan systems. The readout noise does not always scale in a well-behaved way with frequency. The techniques required for higher speed operation have been examined.

\subsection{Wavefront Sensors - for Gemini and UK AO}

We have been involved in analyzing the requirements for two major new projects. The UK is embarking on a project to provide AO systems on its WHT and UKIRT telescopes; detailed atmospheric site monitoring will be followed by design and construction of suitable AO systems. The RGO is also responsible for a Gemini workpackage which will lead to the construction of an $A \& G$ unit for the 8-m telescopes. This unit will have a set of sophisticated wavefront sensors and acquisition facilities.

\section{ACKNOWLEDGMENTS}

We are pleased to acknowledge the support of many RGO colleagues, particularly Dave Banks, John Churchill, Derek Ives, Andrew Johnson, John Maclean, and Percy Terry. 


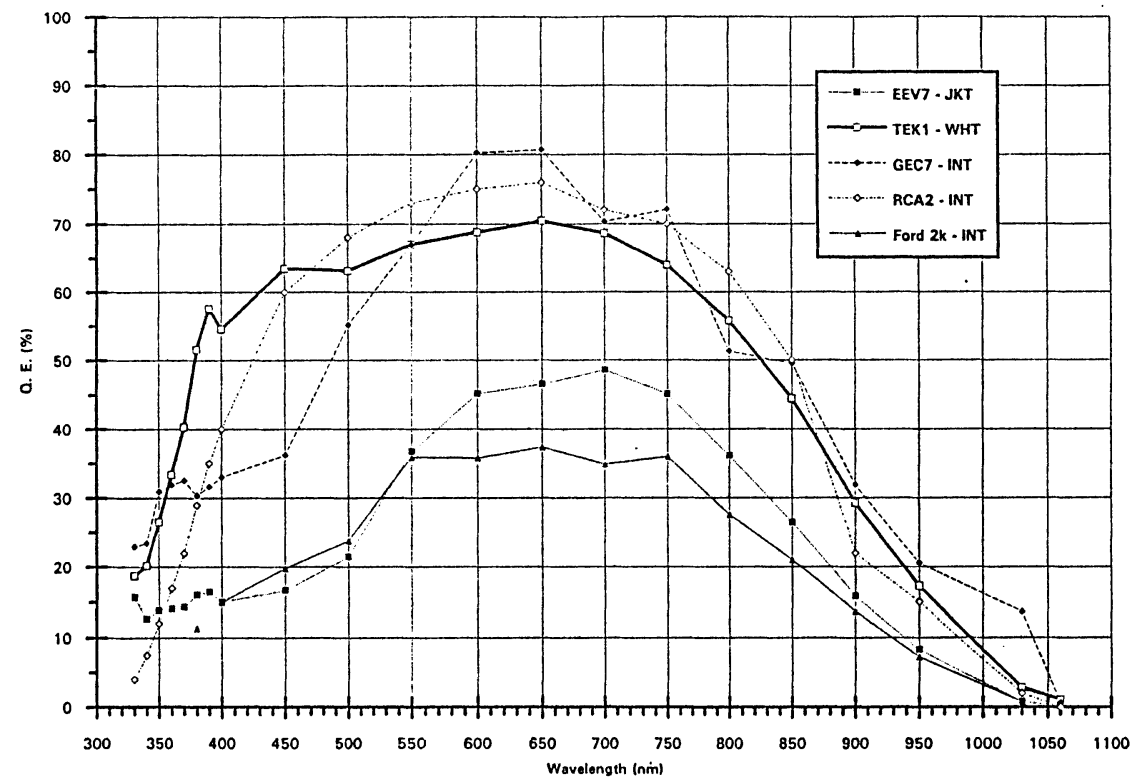

Fig. 2. Spectral response of ING CCD sensors, at cryogenic temperature.

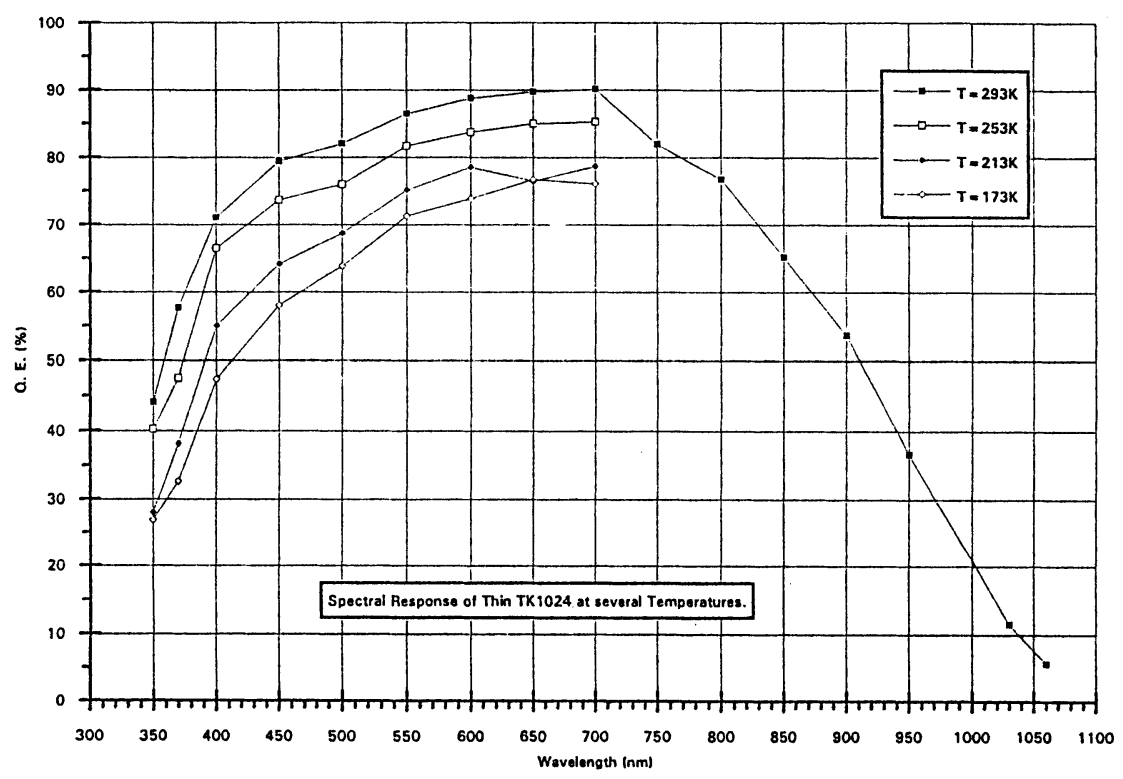

Fig. 3. Variation of spectral response (of thin TK1024 CCD) with temperature. 


\section{REFERENCES}

Bingham, R. G., Gellatly, D. W., Jenkins, C. R., Worswick, S. P. 1994 proc. SPIE 2198, 56

Burke, B. E., Gajar, S. A. 1991 IEEE Trans. Electron Devices 38, 285

Burke, B. E., Mountain, R. W., Harrison, D. C., Bautz, M. W., Doty, J. P., Ricker, G. R. and Daniels, P. J. 1991 IEEE Trans. Electron Devices 38, 1069

Chandler, E.C ., Bredthauer, R. A., Janesick, J. R., Westphal, J. A. and Gunn, J. E. 1990 Proc. SPIE 1242.

Geary, J. and Luppino, G. A. 1994 Proc. SPIE 2201, 588

Holland, A., Turner, M. J. L., Wells, A. and Burt, D. J. 1992 Proc ESA Symposium on Photon Detectors for Space, ESA-SP-356.

Jorden, P. R., Deltorn, J-M. and Oates, A. P. 1994 Proc. SPIE 2198, 836

Lesser M.P., 1994 Proc SPIE 2198, 782.

Luppino, G. A. 1995 in IAU Symposium No. 167, New Developments in Array Technology and Applications, A. G. D. Philip, K. A. Janes and A. R. Upgren, eds., Kluwer Academic Pub. Dordrecht, p 297

Neely, A. W. and Janesick, J. R. 1993 PASP 105, 1330

Stover, R., Wei, M., Gilmore, K. and Brown, W. 1995 in IAU Symposium No. 167, New Developments in Array Technology and Applications, A. G. D. Philip, K. A. Janes and A. R. Upgren, eds., Kluwer Academic Pub. Dordrecht, p 19

Thorne, D. J., Waltham, N. R., Newton, G. M., van Breda, I. G., Fisher, M. and Rudd, P. J. 1990 Proc. SPIE 1235, 400

\section{DISCUSSION}

STOVER: Do you know of a commercial source for small, thinned CCDs suitable for autoguiders?

JORDEN: EEV has a $600 \times 400$ CCD which is used in RGO autoguiders. We have not felt any necessity for smaller formats - in fact we use the full $400 \times 600$ formal to acquire quide stars.

STOVER: Have you found that using "wobble clocks" increased read noise due to extra generated electrons?

JORDEN: This technique is used in the autoguiders at RGO. In cryogenically cooled CCDs this should not be a problem because the clock switching needs to occur only once every few minutes.

STOVER: Do you have some ideas concerning the source of inter-pixel sensitivity variations in the thinned (TEK) CCD?

JORDEN: We were surprised to detect a response modulation (one\%) from thinned (TEK) CCDs. We believe it is a result of the influence of the depletion region reaching to the backside of the chip and influencing charge collection (see reference: Jorden et al. 1994, Proc SPIE 2198, 836).

BLECHA: You mentioned 18 bit converters. In fact we do not need such precision since for high signal the shot noise dominates. Can you comment on the use of non-linear encoding 
scheme?

JORDEN: LOG-converters are very slow. An 18 bit linear converter would be the obvious system upgrade. They would provide a straightforward solution to extending the dynamic range. I agree that full precision is not needed in the presence of shot-noise.

WEAVER: Zoran Ninkov at RIT has reported non-linearities below $1500 \mathrm{e}^{-}$in Kodak CCDs. Have you heard of similar problems with other chips? Do the engineers routinely check for this before releasing them to astronomers?

JORDEN: No. That $1500 \mathrm{e}^{-}$level is very low. We always check our linearity over the whole operating range before installing CCDs on the telescope. 